\title{
Does the Universe Really Expand, or Does the Size of Matter Shrink Instead?
}

\author{
Hwei-Jang Yo \\ Department of Physics, National Cheng-Kung University, Taiwan, China \\ Email: hjyo@phys.ncku.edu.tw
}

How to cite this paper: Yo, H.-J. (2017) Does the Universe Really Expand, or Does the Size of Matter Shrink Instead? Journal of Modern Physics, 8, 2077-2086. https://doi.org/10.4236/jmp.2017.812126

Received: October 18, 2017

Accepted: November 27, 2017

Published: November 30, 2017

Copyright $\odot 2017$ by author and Scientific Research Publishing Inc. This work is licensed under the Creative Commons Attribution International License (CC BY 4.0).

http://creativecommons.org/licenses/by/4.0/

\begin{abstract}
Modern cosmology is built on the concept of the spatial expansion of the Universe. And the current astronomical observation is consistent with this paradigm. However, the expansion of the Universe is an expansion relative to matter. Therefore, in this work we try to switch the viewpoint from the spatial expansion of the Universe to the shrinkage of the size of matter during the evolution of the Universe, by employing Einstein's general relativity and performing a conformal transformation of the metric. The effect of the size shrinkage of matter is then through the variation of the physical parameters/constants in a coordinated way. From this alternative viewpoint, there are advantages in realizing the evolution of the Universe, and also in better understanding Dirac's large number hypothesis.
\end{abstract}

\section{Keywords}

Shrinking Matter, Varying Constant, Large Number Hypothesis

\section{Introduction}

Based on the astronomical observations, besides the Universe being composed of mostly dark matter and dark energy with only $4 \%$ ordinary matter [1], modern cosmology describes the evolution of the Universe as being expanding [2] after its big bang [3] and a cosmic inflation [4]. And the expansion rate of the Universe is increasing with time [5] [6].

Although this achievement in modern cosmology is so tremendous, there still exist many interesting questions to be answered. One of them is the concept of the Universe's spatial expansion. There could be two possible ways in realizing the spatial expansion of the Universe: One is that the Universe does expand spatially while the size of matter stays unchanged, and this is what we usually pic- 
ture; the other is that the Universe keeps its space held constant while the size of matter shrinks. The latter one has ever been considered before. Eddington discussed the possibility of "the shrinking atom" in his book [7]; Wetterich has ever considered in his work [8] a scalar-tensor model in which the size of atom, as well as the Universe, shrinks with its mass increasing. However, there is still a lack of serious and detailed illustration on this possibility from the standard theory-general relativity-without any new physics input. And the reason might originate from its foreseeable complication once the size of matter is linked to the evolution of the Universe.

In this work, we try to explore this possibility of and the mechanism for the size shrinkage of matter during the evolution of the Universe. We find that this paradigm is not only possible but can also be realized with the variation of the physical constants. This idea might remind people of the already existing scalar-tensor theories, especially the Brans-Dicke theory [9] in which the gravitational constant usually varies with a scalar field. Nevertheless, in this work we will mainly stick to the standard general relativity instead of the modified gravity theories. The result will show that, besides the gravitational constant, all the physical constants with the dimensionality of time should vary in a coordinated way such that the Universe still looks expanding in the shrinkage paradigm while all the physical laws remain intact.

The rest of this work is organized as follows: In the next section, we will give a brief description of general relativity applied to modern cosmology; We then conformally transform the metric $g_{\mu \nu}$ into $\bar{g}_{\mu v}$ and demonstrate that these two metrics describe the same evolution of the Universe in Section 3; In Section 4 , the variation of the physical "constants" in the conformal frame, thus in the shrinkage paradigm, are showed; The evolution of the Universe with the shrinkage paradigm and its relation with Dirac's large number hypothesis are described in Section 5. And the conclusion will be presented in the Section 6.

\section{Standard Treatment}

Let us start with a brief description of the general relativity applied to modern cosmology. In Einstein's general relativity, the connection with respect to the metric $g_{\mu v}$ is defined as

$$
\Gamma_{\sigma V}^{\mu}=\frac{1}{2} g^{\mu \lambda}\left(\partial_{\sigma} g_{\lambda v}+\partial_{\nu} g_{\lambda \sigma}-\partial_{\lambda} g_{v \sigma}\right),
$$

and the Ricci tensor is defined as

$$
R_{\mu \nu}=\Gamma_{\mu \nu, \sigma}^{\sigma}-\Gamma_{\mu \sigma, v}^{\sigma}+\Gamma_{\lambda \sigma}^{\sigma} \Gamma_{\mu \nu}^{\lambda}-\Gamma_{\lambda \nu}^{\sigma} \Gamma_{\mu \sigma}^{\lambda} .
$$

Its action is $S=\int \mathscr{\int} \mathrm{d}^{4} x$ with

$$
\mathscr{L}=\mathscr{L}_{\mathrm{g}}+\mathscr{L}_{\mathrm{m}},
$$

and $\mathscr{L}_{g}$ is the Einstein-Hilbert Lagrangian density,

$$
\mathscr{L}_{g}=\frac{1}{2 \kappa} \sqrt{-g} R,
$$


where $\kappa=\frac{8 \pi \stackrel{\circ}{\dot{\circ}^{4}}}{\dot{C}^{4}}$ and the scalar curvature $R=g^{\mu v} R_{\mu v}$. Here we put a circle on the top of a physical constant, e.g., $\stackrel{\circ}{G}$ or $\stackrel{\circ}{c}$, to indicate that it is a true constant or the constant part of the related quantity. And the matter Lagrangian density

$$
\mathscr{L}_{m}=\sqrt{-g} \mathscr{L}_{m}^{\prime}\left(\Phi, g_{\mu v}\right) \text {, }
$$

where $\Phi$ is the matter field. The Euler-Lagrange equation

$$
\frac{\delta \mathscr{L}}{\delta g^{\mu \nu}}=0
$$

leads to Einstein's equation,

$$
G_{\mu \nu}=\kappa T_{\mu \nu}
$$

where the Einstein tensor

$$
G_{\mu \nu} \equiv R_{\mu \nu}-\frac{1}{2} g_{\mu \nu} R
$$

and the stress-energy tensor

$$
T_{\mu \nu} \equiv-\frac{2}{\sqrt{-g}} \frac{\delta \mathscr{L}_{m}}{\delta g^{\mu \nu}} .
$$

With the assumption of homogeneity and isotropy, for the Friedman-Lemaitre-Robertson-Walker (FLRW) metric, the interval ${ }^{1}$ in spherical coordinates $(t, r, \theta, \phi)$ is

$$
\mathrm{d} s^{2}=-\dot{c}^{2} \mathrm{~d} t^{2}+a^{2}(t)\left[\mathrm{d} r^{2}+r^{2}\left(\mathrm{~d} \theta^{2}+\sin ^{2} \theta \mathrm{d} \phi^{2}\right)\right],
$$

thus the nonvanishing metric components are

$$
g_{t t}=-\dot{c}^{2}, g_{r r}=a^{2}, g_{\theta \theta}=a^{2} r^{2}, g_{\phi \phi}=a^{2} r^{2} \sin ^{2} \theta,
$$

and the nonvanishing Einstein tensor components are

$$
G_{t}^{t}=-\frac{3 H^{2}}{\dot{c}^{2}}, \quad G_{r}{ }^{r}=G_{\theta}{ }^{\theta}=G_{\phi}^{\phi}=-\frac{2 \partial_{t} H+3 H^{2}}{\dot{c}^{2}},
$$

where $H \equiv \partial_{t} \ln a$, assuming $a>0$. For a perfect fluid,

$$
T^{\mu v}=\left(\rho \check{c}^{2}+p\right) u^{\mu} u^{v}+p g^{\mu v},
$$

where the 4 -velocity $u^{\mu}=\left(1 / \check{c}^{\circ}, 0,0,0\right)$ and $u_{\mu} u^{\mu}=-1$. So

$$
T_{t}^{t}=-\rho \dot{c}^{2}, \quad T_{r}^{r}=T_{\theta}^{\theta}=T_{\phi}^{\phi}=p .
$$

Einstein's equation thus leads to

$$
3 H^{2}=\kappa \rho \dot{c}^{4}, \quad 2 \dot{H}+3 H^{2}=-\kappa p \dot{c}^{2} .
$$

And it gives

$$
H \propto a^{-3(1+w) / 2},
$$

for the equation of state $p=w \rho c^{2}$ with $w=0$ standing for the matter-dominated ${ }^{1}$ Here we only consider the zero spatial curvature case, i.e., $k=0$, since the conclusion in this work will remain the same even in the $k= \pm 1$ cases. 
universe case, $w=1 / 3$ for the radiation-dominated universe case, and $w=-1$ for the cosmological-constant-dominated universe case.

\section{Conformal Treatment}

Now let us switch our point of view by using a conformal transformation from $g_{\mu \nu}$ to $\bar{g}_{\mu v}$ with $g_{\mu \nu}=e^{\psi} \bar{g}_{\mu \nu}$, then the relation between the Ricci tensors is

$$
R_{\mu \nu}=\bar{R}_{\mu \nu}-\bar{\nabla}_{\mu} \bar{\nabla}_{\nu} \psi-\frac{1}{2} \bar{g}_{\mu \nu} \bar{\nabla}^{2} \psi+\frac{1}{2} \bar{\nabla}_{\mu} \psi \bar{\nabla}_{\nu} \psi-\frac{1}{2} \bar{g}_{\mu \nu}(\bar{\nabla} \psi)^{2},
$$

where $\bar{\nabla}_{\mu}$ is the covariant derivative with respect to the conformal metric $\bar{g}_{\mu v}$, and $\bar{\nabla}^{2} \psi$ and $(\bar{\nabla} \psi)^{2}$ are the shorthands of $\bar{g}^{\mu \nu} \bar{\nabla}_{\mu} \bar{\nabla}_{\nu} \psi$ and $\bar{g}^{\mu \nu} \bar{\nabla}_{\mu} \psi \bar{\nabla}_{\nu} \psi$ respectively.

Since $\sqrt{-g}=e^{2 \psi} \sqrt{-\bar{g}}$, and

$$
R=e^{-\psi}\left[\bar{R}-3 \bar{\nabla}^{2} \psi-\frac{3}{2}(\bar{\nabla} \psi)^{2}\right],
$$

so the Einstein-Hilbert Lagrangian density becomes

$$
\begin{aligned}
\mathscr{L}_{g} & =\frac{1}{2 \kappa} \sqrt{-g} R=\frac{e^{\psi}}{2 \kappa} \sqrt{-\bar{g}}\left[\bar{R}-3 \bar{\nabla}^{2} \psi-\frac{3}{2}(\bar{\nabla} \psi)^{2}\right] \\
& \simeq \frac{e^{\psi}}{2 \kappa} \sqrt{-\bar{g}}\left[\bar{R}+\frac{3}{2}(\bar{\nabla} \psi)^{2}\right],
\end{aligned}
$$

where the symbol $\simeq$ means that the total derivative term is ignored after integrating by parts the second derivative term into one first derivative quadratic term and a total derivative term, and the matter Lagrangian density becomes

$$
\mathscr{L}_{m}=\sqrt{-g} \mathscr{L}_{m}^{\prime}=e^{2 \psi} \sqrt{-\bar{g}} \mathscr{L}_{m}^{\prime}\left(\Phi, e^{\psi} \bar{g}_{\mu v}\right) .
$$

Therefore, the total Lagrangian density becomes

$$
\mathscr{L}=\sqrt{-\bar{g}}\left[\frac{e^{\psi}}{2 \kappa}\left(\bar{R}+\frac{3}{2}(\bar{\nabla} \psi)^{2}\right)+e^{2 \psi} \mathscr{L}_{m}^{\prime}\right] .
$$

Here we encounter some difficulty. The second term in the RHS of Equation (21) has a "wrong sign" which makes its kinetic term become negative. Such a ghost term is usually disliked by theorists because it carries negative energy, although some theories allow its existence [10]. However, this issue in general relativity has long been identified in [11] [12] that $\psi$ is related to the (global) timelike variable, with a negative hyperbolic signature. We can understand it as follows: Gravity is purely attractive and hence configurations are inherently physically unstable to expansion/contraction. And the sign of this term just reflects this unstable physical mode of gravitational dynamics. Since it is a characteristic of general relativity, we will not avoid this term by any modification during the comparison between the one with $g_{\mu v}$ and the other with the conformal metric $\bar{g}_{\mu \nu}$. Besides, it should do no harm within the familiar FLRW metric. After all, all we have done is simply a conformal transformation of the metric. We will come back to this point later. 
The Euler-Lagrange equations for $\bar{g}^{\mu v}$, i.e.,

$$
\frac{\delta \mathscr{L}}{\delta \bar{g}^{\mu \nu}}=0 \text {, }
$$

gives

$$
\begin{gathered}
\frac{\delta \mathscr{L}_{g}}{\delta \bar{g}^{\mu \nu}}=\frac{e^{\psi}}{2 \kappa} \sqrt{-\bar{g}}\left[\bar{G}_{\mu \nu}+\bar{g}_{\mu \nu} \bar{\nabla}^{2} \psi-\bar{\nabla}_{\mu} \bar{\nabla}_{\nu} \psi+\frac{1}{2} \bar{\nabla}_{\mu} \psi \bar{\nabla}_{\nu} \psi+\frac{1}{4} \bar{g}_{\mu \nu}(\bar{\nabla} \psi)^{2}\right] \\
\frac{\delta \mathscr{L}_{m}}{\delta \bar{g}^{\mu \nu}}=e^{2 \psi} \sqrt{-\bar{g}}\left(\frac{\delta \mathscr{L}_{m}^{\prime}}{\delta \bar{g}^{\mu \nu}}-\frac{1}{2} \bar{g}_{\mu \nu} \mathscr{L}_{m}^{\prime}\right)=-\frac{\sqrt{-\bar{g}}}{2} \bar{T}_{\mu \nu} .
\end{gathered}
$$

By comparing the expressions, it can be showed $\bar{T}_{\mu v}=e^{\psi} T_{\mu v}$. And $\bar{T}_{v}^{\mu}=e^{2 \psi} T^{\mu}$. Thus

$$
\bar{G}^{\mu}{ }_{v}-\bar{\nabla}^{\mu} \bar{\nabla}_{v} \psi+\delta^{\mu}{ }_{v} \bar{\nabla}^{2} \psi+\frac{1}{2} \bar{\nabla}^{\mu} \psi \bar{\nabla}_{v} \psi+\frac{1}{4} \delta^{\mu}{ }_{v}(\bar{\nabla} \psi)^{2}=\frac{\kappa}{e^{\psi}} \bar{T}^{\mu}{ }_{v}=\kappa e^{\psi} T^{\mu}{ }_{v} .
$$

The interval for the FLRW metric in Equation (10) can be expressed as

$$
\begin{aligned}
\mathrm{d} s^{2} & =g_{\mu \nu} \mathrm{d} x^{v} \mathrm{~d} x^{v}=e^{\psi} \bar{g}_{\mu \nu} \mathrm{d} x^{\mu} \mathrm{d} x^{v} \\
& =e^{\psi}\left[-\frac{\stackrel{\circ}{C}^{2}}{e^{\psi}} \mathrm{d} t^{2}+\mathrm{d} r^{2}+r^{2}\left(\mathrm{~d} \theta^{2}+\sin ^{2} \theta \mathrm{d} \phi^{2}\right)\right],
\end{aligned}
$$

where we set

$$
e^{\psi}=a^{2}(t)
$$

and the nonvanishing conformal metric components are

$$
\bar{g}_{t t}=-\frac{\grave{c}^{2}}{a^{2}}, \bar{g}_{r r}=1, \bar{g}_{\theta \theta}=r^{2}, \bar{g}_{\phi \phi}=r^{2} \sin ^{2} \theta .
$$

Under this conformal transformation, it turns out that the conformal Ricci tensor vanishes, i.e., $\bar{R}_{\mu v}=0$. So does the conformal Einstein tensor, $\bar{G}_{\mu v}=0$. Then, with $\psi=2 \ln a$, it is easy to show that Equation (25) is able to be converted to the familiar Equation (15). Therefore, the Lagrangian density (21) with the conformal metric $\bar{g}_{\mu v}$ describes exactly the same physics as the Lagrangian density with $g_{\mu \nu}$ does, without any surprise.

For $\psi$, the Euler-Lagrange equations

$$
\frac{\delta \mathscr{L}}{\delta \psi}=0,
$$

leads to

$$
\bar{\nabla}^{2} \psi+\frac{1}{2}(\bar{\nabla} \psi)^{2}=\frac{\bar{R}}{3}+\frac{\kappa}{3} e^{-\psi} \bar{T} .
$$

Equation (30) is the contraction of Equation (25). This simply shows that these two equations are not independent. With the conformal metric (28), Equation (30) becomes

$$
6 \dot{H}+12 H^{2}=-\kappa T \dot{c}^{2},
$$

which is basically a combination of Equation (15). 


\section{Varying Constants}

After demonstrating the equivalence of using either $g_{\mu \nu}$ or the conformal one $\bar{g}_{\mu v}$ for evolving the Universe, we can now turn back to the viewpoint switching. From the Einstein-Hilbert Lagrangian density to the one (21), one can easily recognize that the viewpoint is switched from the Einstein frame to the Jordan frame [13]. It has been a debate for a long time on one's superiority to the other. Here we are only interested in their equivalence for describing physics.

The interval (26) can be written as

$$
\mathrm{ds} s^{2}=a^{2} \mathrm{~d} \bar{s}^{2}
$$

and the conformal interval $\mathrm{d} \bar{s}^{2}$ can be expressed as

$$
\mathrm{d} \bar{s}^{2}=-c^{2} \mathrm{~d} t^{2}+\mathrm{d} r^{2}+r^{2}\left(\mathrm{~d} \theta^{2}+\sin ^{2} \theta \mathrm{d} \phi^{2}\right),
$$

where

$$
c(t) \equiv \frac{\stackrel{\circ}{C}}{a(t)}=\frac{\stackrel{\circ}{c}}{e^{\psi / 2}} .
$$

Then the total Lagrangian density (21) can be rewritten as

$$
\begin{aligned}
\mathscr{L} & =\sqrt{-\bar{g}}\left[\frac{\dot{c}^{4} e^{\psi}}{16 \pi \dot{G}}\left(\bar{R}+\frac{3}{2}(\bar{\nabla} \psi)^{2}\right)+e^{2 \psi} \mathscr{L}_{m}^{\prime}\right] \\
& =\frac{\dot{c}^{4}}{16 \pi G} \sqrt{-\bar{g}}\left[\bar{R}+\frac{3}{2}(\bar{\nabla} \psi)^{2}\right]+\frac{\dot{c}^{4}}{c^{4}} \sqrt{-\bar{g}} \mathscr{L}_{m}^{\prime},
\end{aligned}
$$

where

$$
G(t) \equiv \frac{\stackrel{\circ}{\mathrm{G}}}{e^{\psi}}=\frac{\stackrel{\circ}{G}}{a^{2}} .
$$

In Equation (35), the first term in the RHS, i.e., $\frac{{ }^{\circ} 4}{16 \pi G} \sqrt{-\bar{g}} \bar{R}$, can be regarded as the Einstein-Hilbert Lagrangian density with the conformal metric $\bar{g}_{\mu \nu}$, the last term in the RHS, i.e., $\frac{\dot{c}^{4}}{c^{4}} \sqrt{-\bar{g}} \mathscr{L}_{m}^{\prime}$, is the matter Lagrangian density, while the middle term in the RHS, i.e., $\frac{3}{2} \frac{\stackrel{\circ}{C}^{4}}{16 \pi G} \sqrt{-\bar{g}}(\bar{\nabla} \psi)^{2}$, is responsible for the dynamical behavior of $\psi$ (and thus $a$ ) after $a^{2}$ is extracted out of $g_{\mu v}$ in the conformal transformation.

If we take $G$ and $c$ as the gravitational "constant" and the speed of light respectively in the shrinkage paradigm although they vary with respect to time via $a(t)$, then, by using them, we can equivalently explain the astronomical data without employing the concept of the spatial expansion of the Universe. This can be understood from checking the conformal interval (33) in which the spatial part does not change with time. In the other words, instead of the Universe expanding with the size of matter unchanged, here we consider the Universe intact but the size of matter shrinking. And the shrinkage of matter is through the varying/decreasing of the physical constants, e.g., $G$ and $c$. 
Of course it is unacceptable to change any physical law without compelling reasons under the current situation. Therefore, we can instead apply the known $a$-dependence of the constants to physical laws to obtain the $a$-dependent behavior of the other physical quantities. For example, for the electromagnetic wave,

$$
c=\text { wavelength } \times \text { frequency. }
$$

Within the shrinkage paradigm, wavelength is a spatial distance and thus unchanged during the evolution of the Universe, and $c \propto a^{-1}$, therefore this leads to

$$
\text { frequency } \propto a^{-1} \text {. }
$$

To make the Planck-Einstein relation work, we have

$$
E=\gamma m_{0} c^{2}=h v,
$$

where $v$ is the related frequency. The rest mass $m_{0}$ is about matter and the Lorentz factor $\gamma$ is dimensionless. Thus they will stay unchanged. Therefore it is easy to obtain the a-dependence of the Planck constant

$$
h \propto a^{-1}
$$

which indicates that the Planck constant will decrease as long as a increases with respect of time. We then have the $a$-dependence of the three fundamental physical constants most employed in general relativity and cosmology,

$$
G \propto a^{-2}, \quad c \propto a^{-1}, \quad h \propto a^{-1} .
$$

Note that the order of the a-dependence of the physical constants is the same as the order of their dimensionality of time. And

$$
\sqrt{\frac{G}{G_{\text {now }}}}=\frac{c}{c_{\text {now }}}=\frac{h}{h_{\text {now }}}=\frac{a_{\text {now }}}{a}=1+z,
$$

where $z$ is the so-called cosmological redshift ${ }^{2}$. One can easily deduce the $a$ -dependence of the other physical quantities/constants by applying the known dependence, e.g., the ones in Equation (41), to the related physical laws.

There might be concerns on the details of the Universe's evolution along with the shrinkage paradigm, e.g., big bang nucleosynthesis, cosmic inflation, inhomogeneity and perturbation, cosmological constant, etc. As we have emphasized in the context, the shrinkage paradigm is only an alternative viewpoint on the Universe's evolution, compared with the expansion paradigm. Both of the paradigms should give either the same or the equivalent physical result.

\section{Discussion}

The (classical) scenario for the evolution of the Universe with the shrinkage

${ }^{2}$ In the shrinkage paradigm, the meaning of $z$ is different from the cosmological redshift in the expansion paradigm. However, its form of definition and value are the same in the two paradigms. 
paradigm is as follows: At the beginning of our Universe, $a \rightarrow 0$, thus all the $a$-dependent physical quantities approached to their extreme values, e.g., infinity or zero. It made all the related physical interactions become extremely strong in the Universe such that the effect from the spatial distance between matter was relatively extremely small compared with the coupling strengths of the interactions. This corresponds the so-called singular point of the Universe. Then the explosive change of $a$ leaded to the explosive changes of these $a$-dependent quantities. It corresponds to the big-bang period in cosmology. This led to the weakening of the strength of the physical interactions between matter. And the weakening made the Universe "become larger and expand" from the viewpoint of matter. There was a period of time when a increased exponentially. This leaded the $a$-dependent physical quantities to increase/decrease exponentially-the cosmic inflation. Currently $a$ is again increasing acceleratingly and this corresponds to the accelerating expansion of the Universe in cosmology. Nevertheless, the spatial size of the Universe never changes from the big-bang to the current time with the shrinkage paradigm.

The following is one example that one could understand better and appreciate a controversial theory when it is reviewed with the shrinkage paradigm. In Dirac's large number hypothesis [14] [15] [16], he suggested that all large numbers obtained by combining the fundamental atomic constants and cosmic parameters must be related, and this implied a deeper meaning of Nature. The consequence of his hypothesis is that the gravitational constant should change with cosmic time, i.e.,

$$
G \sim t^{-1}
$$

However, Dirac's predictions have never been favored by modern cosmology. Here we are able to address the constant variation issue with the viewpoint of the shrinkage paradigm. With this paradigm, it is natural for $G$ to vary with time. However, we have instead

$$
G \propto a^{-2} \sim f(t),
$$

where $f(t)=t^{-1}$ in the radiation-dominated era, $f(t)=t^{-4 / 3}$ in the matter-dominated era, and $f(t)=e^{-\mid \text {constant|xt }}$ in the cosmological constant era ${ }^{3}$. We check the large number from the ratio of the electromagnetic force to the gravitational force between a proton and an electron and find that the ratio remains constant during the evolution of the Universe,

$$
\frac{F_{\mathrm{EM}}}{F_{\text {gravity }}}=\frac{e^{2}}{4 \pi \epsilon_{0} G m_{p} m_{e}}=\text { constant, }
$$

since only the vacuum permittivity $\epsilon_{0}$ and $G$ in the expression vary with time but

$$
\epsilon_{0} \propto a^{2}
$$

${ }^{3}$ The assumptions behind Dirac's derivation are not exactly as same as the ones in the shrinkage paradigm. Here we only compare the final result. 
due to $c \propto a^{-1}$, where $m_{p}\left(m_{e}\right)$ is the mass of proton (electron), and $e$ is the electric charge of electron. It will be interesting to check the constancy of all the large numbers for the study of the large number hypothesis in the framework of this paradigm. The benefit within this paradigm is that, by only employing general relativity, without any modification and new physics, we can both explain an expanding universe and, at the same time, realize and appreciate Dirac's legendary theory.

As to the ghost term in Lagrangian (21), we could have a quick fix by adding a term like $-\sqrt{-\bar{g}} \frac{e^{\psi}}{2 \kappa} \sigma(\bar{\nabla} \psi)^{2}$ by hand to Lagrangian (21) with $\sigma-\frac{3}{2}>0$. The addition will turn the kinetic term of $\psi$ in the modified Lagrangian positive and thus get rid of the concern of negative energy. However, the addition makes the theory deviate from general relativity to a scalar-tensor one in which $\psi$ is promoted generically from a global timelike variable to an independent scalar field. It might be interesting to see how far this type of scalar-tensor theory of modified gravity could go in explaining the evolution of the Universe. However, this is beyond the scope of this work.

\section{Conclusion}

In this work, we switch the viewpoint from the spatial expansion of the Universe to the shrinkage of the size of matter during the evolution of the Universe. We find that this shrinkage paradigm can be realized by means of the variation of the physical constants. In spite of its slight complication, this paradigm is equally good with the viewpoint of the Universe's spatial expansion in describing physics. The shrinkage paradigm is advantageous to the expansion one in some special cases, especially, in reviewing the large number hypothesis.

\section{Acknowledgements}

The author thanks James M. Nester, Chopin Soo, and Ronald E. Taam for interesting discussions and helpful comments. This work was supported in part by the Ministry of Science and Technology under Grant No. MOST106-2112-M-006-011.

\section{References}

[1] Jarosik, N., et al. (2011) The Astrophysical Journal Supplement Series, 192, 14.

[2] Hubble, E. (1929) Proceedings of the National Academy of Sciences of the United States of America, 15, 168-173. https://doi.org/10.1073/pnas.15.3.168

[3] Alpher, R.A., Bethe, H. and Gamow, G. (1948) Physical Review Letters, 73, 803.

[4] Guth, A. (1981) Physical Review D, 23, 347.

[5] Riess, A.G., et al. (1998) The Astronomical Journal, 116, 1009.

[6] Schmidt, B.P., et al. (1998) Astrophysical Journal, 507, 46.

[7] Eddington, A.S. (1933) The Expanding Universe. University Press, Cambridge. https://doi.org/10.1017/CBO9780511564208

[8] Wetterich, C. (2013) Physics of the Dark Universe, 2, 184-187. 
https://doi.org/10.1016/j.dark.2013.10.002

[9] Brans, C.H. and Dicke, R.H. (1961) Physical Review, 124, 925.

https://doi.org/10.1103/PhysRev.124.925

[10] Fujii, Y. and Maeda, K. (2004) The Scalar-Tensor Theory of Gravitation. Cambridge University Press, Cambridge. https://doi.org/10.1017/CBO9780511535093

[11] DeWitt, B.S. (1967) Physical Review, 160, 1113.

https://doi.org/10.1103/PhysRev.160.1113

[12] Ó Murchadha, N. and York, Jr., J.W. (1974) Physical Review D, 10, 428.

https://doi.org/10.1103/PhysRevD.10.428

[13] Jordan, P. (1959) Zeitschrift für Physik, 157, 112-121.

https://doi.org/10.1007/BF01375155

[14] Dirac, P.A.M. (1937) Nature, 139, 323.

[15] Cetto, A., de la Peña, L. and Santos, E. (1986) Astronomy \& Astrophysics, 164, 1.

[16] Ray, S., Mukhopadhyay, U. and Ghosh, P.P. (2007) arXiv:0705.1836. 